\title{
Hygiene Hypothesis Indicators and Prevalence of Antinuclear Antibodies in US Adolescents
}

\author{
Helen C. S. Meier ${ }^{1 *}$, Dale P. Sandler ${ }^{2}$, Jesse Wilkerson ${ }^{3}$, Frederick W. Miller ${ }^{4}$, \\ Gregg E. Dinse ${ }^{3}$ and Christine G. Parks ${ }^{2}$
}

${ }^{1}$ Population, Neurodevelopment and Genetics Program, Survey Research Center, Institute for Social Research, University of Michigan, Ann Arbor, MI, United States, ${ }^{2}$ Epidemiology Branch, National Institute of Environmental Health Sciences (NIEHS), Durham, NC, United States, ${ }^{3}$ Public Health \& Scientific Research, Social and Scientific Systems, Durham, NC, United States, ${ }^{4}$ Environmental Autoimmunity Group, National Institute of Environmental Health Sciences (NIEHS), Durham, NC, United States

\section{OPEN ACCESS}

Edited by:

Nancy Agmon-Levin, Sheba Medical Center, Israel

Reviewed by: Elham Hossny, Ain Shams University, Egypt Marvin Fritzler,

University of Calgary, Canada

*Correspondence: Helen C. S. Meier hspink@umich.edu

Specialty section: This article was submitted to Autoimmune and Autoinflammatory Disorders, a section of the journal

Frontiers in Immunology

Received: 04 October 2021 Accepted: 07 January 2022 Published: 28 January 2022

Citation: Meier HCS, Sandler DP, Wilkerson J, Miller FW, Dinse GE and Parks CG

(2022) Hygiene Hypothesis Indicators and Prevalence of Antinuclear Antibodies in US Adolescents.

Front. Immunol. 13:789379. doi: 10.3389/fimmu.2022.789379
Autoimmunity prevalence, as measured by antinuclear antibodies (ANA), is increasing in U.S. adolescents. Improved hygiene and cleaner environments in childhood may reduce exposure to infections and other immune challenges, resulting in improper immune responses to later-life exposures. We examined associations of hygiene hypothesis indicators, including asthma, allergies, and antibodies to infectious agents, with ANA prevalence, measured by HEp-2 immunofluorescence, in adolescents (aged 12-19 years) over a 25-year time span in the National Health and Nutrition Examination Survey (NHANES) $(\mathrm{N}=2,709)$, adjusting for age, sex, race/ethnicity, body mass index, education and survey cycle, overall and within individual time periods, using logistic regression. Prevalence of ANA in adolescents increased from 5.0\% in 1988-1991 to $12.8 \%$ in 2011-2012. ANA were positively associated with diagnosis of asthma in early childhood (OR: 2.07, Cl: 1.09-3.99) and the effect estimate for current hay fever was elevated but not statistically significant (OR: 1.55, Cl: 0.85-2.84). Fewer than $2 \%$ of those with ANA in 1988-1991 had been diagnosed with asthma, compared with 18\% in 19992000, and 27\% in 2003-2004 and 2011-2012. ANA trended negatively with Helicobacter pylori antibodies (OR: 0.49, Cl: 0.24-0.99). ANA may be useful as an additional indicator of inadequate immune education in adolescence, a critical period of growth and development.

Keywords: antinuclear antibodies (ANA), hygiene hypothesis, adolescents, asthma, allergy, autoimmunity

\section{INTRODUCTION}

The prevalence of autoimmunity, characterized as the immune system responding to nonthreatening self-antigens, was recently shown in a nationally representative sample of U.S. adolescents to have increased substantially over the last 25 years (1). Drivers of this trend remain unknown (1). Developing immune systems in children and adolescents require education through immune challenges to function properly later in life (2). The hygiene hypothesis posits that

Abbreviations: ANA, antinuclear antibodies; CI, confidence interval; NHANES, National Health and Nutrition Examination Survey; OR, odds ratio. 
improved hygiene and clean environments in childhood lead to declining exposure to infections and other historically common immune challenges and to improper immune responses to nonthreatening exposures, such as allergens later in life (3-6). Indeed, asthma and allergy are commonly used as indicators for inadequate immune challenge in childhood (7) and asthma prevalence in adolescents has also steadily increased over time while the prevalence of common infection indicators has decreased $(8,9)$.

Immune mechanisms underlying the hygiene hypothesis are not fully-established but include the possibility that reduced microbial exposures in early life may prevent the activation of regulatory T-cells, thereby enabling both the development of allergic diseases and activation of autoimmune pathways (4). To elucidate whether autoimmunity, measured by antinuclear antibodies (ANA), may be an additional indicator of inadequate immune system education in childhood and adolescence, we explored age and birth cohort associations of common hygiene hypothesis indicators, including asthma, allergy, and persistent infections, with the presence of ANA in a representative sample of U.S. adolescents from the National Health and Nutrition Examination Survey (NHANES) (Figure 1).

We hypothesized that an increase in allergy and asthma prevalence seen over 25 years in the U.S. would be correlated with the increase in ANA prevalence observed in adolescents. Further, we hypothesized that lower prevalence of common types of infections, would be inversely associated with ANA prevalence.

\section{MATERIALS AND METHODS}

The study population was all adolescents, aged 12-19 years, from four cross-sectional NHANES cycles for which ANA data were available (overall sample, $\mathrm{N}=2,709$ ): 1988-1991 ( $\mathrm{N}=676$ ), 19992000 ( $\mathrm{N}=609), 2003-2004(\mathrm{~N}=581)$ and 2011-2012 ( $\mathrm{N}=843)$. The 2001-2002 cycle was not included as no individuals aged 12-19 were tested for ANA. NHANES is a representative survey of the non-institutionalized U.S. population. Provided subsample specific weights were used to adjust for nonresponse and the probability of selection into each ANA subsample (10). The NHANES protocol was approved by the National Center for Health Statistics (NCHS) Ethics Review Board of the Centers for Disease Control and
Prevention (CDC). All participants or guardians gave written informed consent. Laboratory assays were conducted previously as part of standardized protocols available at: https://wwwn.cdc. gov/nchs/nhanes/Search/DataPage.aspx?Component=Laboratory.

\subsection{Outcome}

ANA were measured across four time periods as described previously (1) with indirect immunofluorescence at a 1:80 dilution using the NOVA Lite HEp-2 ANA slide with DAPI kit (INOVA Diagnostics, San Diego, CA) with a highly specific fluorescein isothiocyanate (FITC)-conjugated secondary antibody (goat anti-human IgG). Images were captured via the NOVA View automated fluorescence microscope system (INOVA Diagnostics) and stored digitally. Staining intensities were graded from 0 to 4 relative to a standard reference gallery, with non-zero values (e.g., values 1-4) considered indicative of ANA positivity (1). All samples from all years were assayed at the same time, using the same methods in a single laboratory. Readings were made independently by at least two experienced evaluators (blinded to sample characteristics and time period), who agreed on $>95 \%$ of the intensities and patterns; differences were resolved by consensus or adjudicated by a third blinded rater. Repeat testing of random samples showed $>98 \%$ concordance.

\subsection{Exposures}

Except where specified, data on exposures were available across all four cycles. Serology methods varied for Toxoplasma and Herpes assays by cycle as described below.

\subsubsection{Asthma}

Participants were asked if a "doctor or other health professional ever told you that you have asthma." Affirmative responders were then asked, "How old were you when you were first told you had asthma?" Responses were categorized as, asthma diagnosed before age 6 , asthma diagnosed at age 6 or later, or no asthma diagnosis (referent). Current asthma was ascertained by asking, "Do you still have asthma?"

\subsubsection{Allergy}

Participants were asked, "During the past 12 months, have you had hay fever?" Responses were categorized as "yes" or "no".

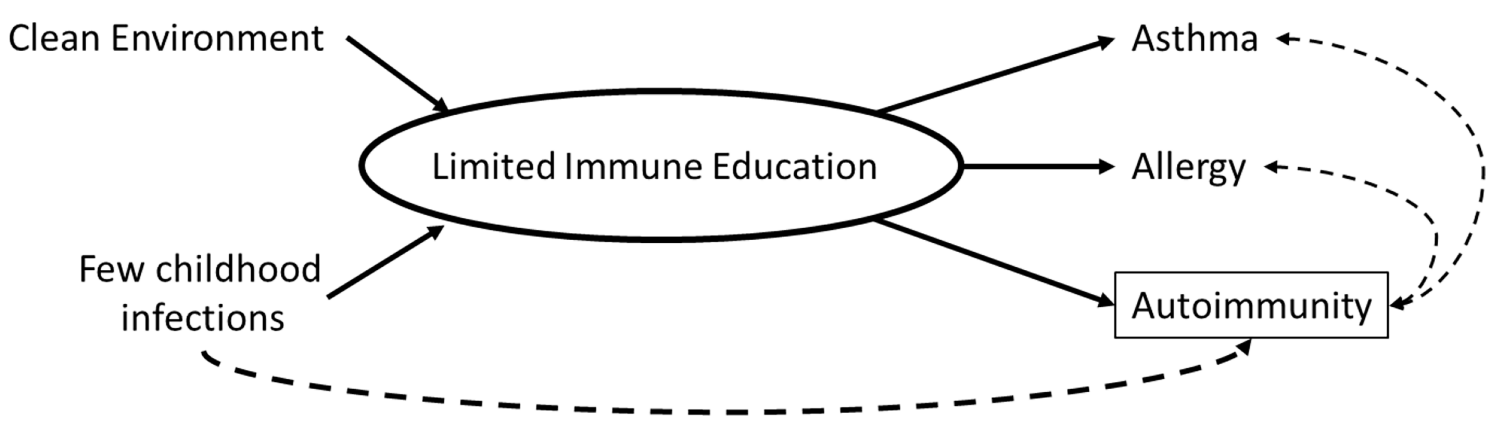

FIGURE 1 | Conceptual diagram of the relationship between hygiene hypothesis indicators and autoimmunity. 
Atopic asthma was derived by combining self-reported current asthma and hay fever status into a four-level categorical variable: no atopy (referent), hay fever only, current asthma only, and both hay fever and current asthma.

\subsubsection{Infections}

\subsubsection{Helicobacter pylori (H. pylori) Seropositivity -} 1988-1991 and 1999-2000 Only

H. pylori antibody testing was previously performed at the University of Washington on surplus sera. H. pylori-specific IgG was measured using Wampole Laboratories (Cranbury, NJ) H. Pylori IgG Enzyme-Linked Immunosorbent Assay (ELISA). Standard ELISA cut-offs were used to categorize participants into seropositive (optical density (OD) value $>1.1$ ) or seronegative (OD value $<0.9$ ) to $H$. pylori. Equivocal values (0.9-1.1) were categorized as seronegative to produce conservative estimates of serostatus.

\subsubsection{Toxoplasma gondii (T. gondii) Seropositivity}

1988-1991: The presence and quantity of antibodies to T. gondii were determined by comparing the OD of the test sample to a standard curve constructed using optical density readings from positive control sera obtained from a kit; these readings were calibrated to WHO Toxo 60 serum and read as International Units $(\mathrm{IU} / \mathrm{mL})$. Test samples with results $\geq 7 \mathrm{IU} / \mathrm{mL}$ were considered to be positive, indicating infection at some undetermined time.

1999-2000 and 2003-2004: IgG to T. gondii was measured using a solid-phase enzyme immunoassay. Using OD readings from positive controls, a standard curve was constructed and calibrated to WHO Toxo 60 serum. Samples with OD $\geq 10 \mathrm{IU} /$ $\mathrm{mL}$ were determined to be positive for $T$. gondii infection.

2011-2012: IgG to T. gondii was measured using an enzyme immunoassay. Samples with $\geq 33 \mathrm{IU} / \mathrm{mL}$ were coded as positive for T. gondii infection.

\subsubsection{Herpes Simplex Virus-1 (HSV-1) Seropositivity}

All time periods (1988-1991, 1999-2000, 2003-2004 and 20112012): Sera from participants aged 14-18 were tested using solidphase enzymatic immunodot assay specific for HSV-1 glycoprotein at Emory University, Atlanta, GA (11). Serum reactive to an immunodot containing $\mathrm{gG}-1$ indicated previous and probable latent HSV-1 infection and was classified as seropositive.

\subsection{Covariates}

Covariates, chosen based on a priori knowledge of their relationships with the exposures and ANA positivity, included age (years), self-reported sex (male or female), race/ethnicity, education of reference parent, and body mass index (BMI). Race/ ethnicity was categorized into Non-Hispanic White, NonHispanic Black, or other. Education of reference parent was categorized as less than high school, high school, or more than high school. BMI categories were calculated using CDC growth curve percentiles and classified as underweight/normal $\left(<85^{\text {th }}\right.$ percentile), overweight $\left(85^{\text {th }}\right.$ to $<95^{\text {th }}$ percentile), or obese $\left(\geq 95^{\text {th }}\right.$ percentile). Tobacco smoke exposure, country of birth, and household size were included in descriptive characterization of ANA status. Tobacco smoke exposure was classified based on serum cotinine levels as smoker $(>15 \mathrm{ng} / \mathrm{mL})$, secondhand exposure (limit of detection-15ng/mL), and no exposure (below the limit of detection). Country of birth was dichotomized as U.S. or other. Household size was categorized as 1-3 individuals, 4-5 individuals, or 6+ individuals.

\subsection{Analyses}

Prevalence of ANA was estimated for each time period both overall and by participant characteristics, including demographics, health status, and infection seropositivity. Unadjusted associations between ANA positivity and each characteristic were assessed via Rao-Scott $\chi^{2}$-tests for categorical characteristics and F-tests for continuous characteristics. Prevalence odds ratios (ORs) and 95\% confidence intervals (CIs) from logistic regression were used to assess associations between ANA and each characteristic within and across all time periods, adjusted for age, sex, race/ethnicity, BMI, and education of reference parent. Sensitivity analyses additionally adjusted for tobacco smoke exposure, country of birth and household size.

Assessment of ANA trend over time was based on two adjusted logistic regression models. The first model added a categorical covariate for time period and estimated the ORs and 95\% CIs for ANA positivity in each period, relative to the first. The second model instead added a quantitative covariate for the number of years between period midpoints, relative to the first $(0,10,14$, and 22 years $)$, and produced a P-value from a $\chi^{2}$-test to assess an ANA positivity time trend. Both time trend logistic regression models were each adjusted for age, sex, race/ethnicity, BMI, and education of reference parent. All analyses were performed using SAS software survey procedures (version 9.4; SAS Institute, Cary, NC). Sampling weights and design variables were incorporated in all analyses to obtain unbiased national prevalence and variance estimates.

\section{RESULTS}

\subsection{Cross-Sectional Analyses by Time Period}

The percent of ANA positive adolescents increased over time from $5.0 \%$ in $1988-1991$ to $12.8 \%$ in $2011-2012$ (Figure 2 ). The prevalence of asthma also increased over that time span, while the prevalence of infection indicators, such as antibodies to H.pylori, T. gondii, and HSV-1, has steadily decreased over time.

The distributions of participant characteristics by ANA status for each time period are depicted in Table 1. Within most time periods, ANA positive individuals were more likely to be female, but no other associations between ANA and demographic characteristics emerged consistently across time periods. ANA were not patterned by age, race/ethnicity, education, or smoking. Several characteristics exhibited associations with ANA in specific time periods. In 2003-2004, ANA positive individuals were less likely to be obese than ANA negatives and ANA 


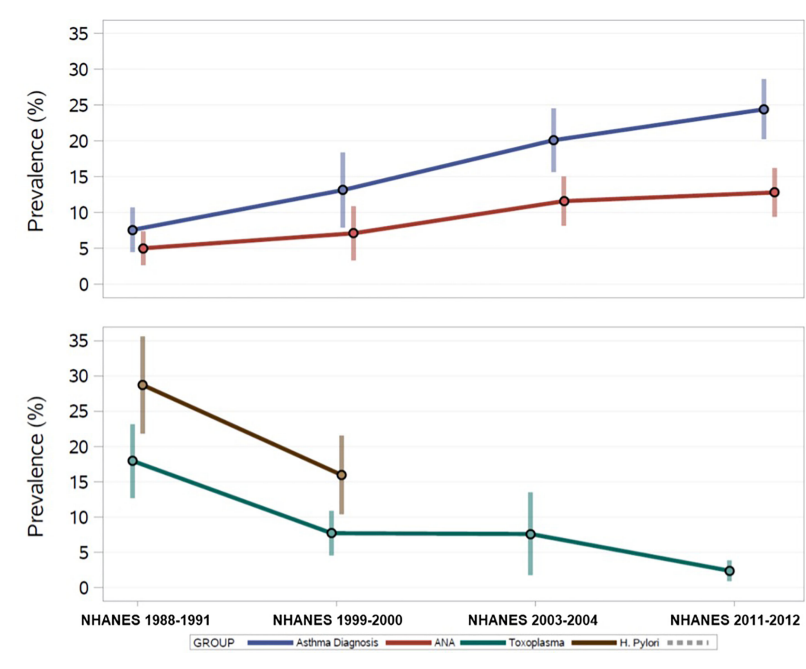

FIGURE 2 | U.S. Prevalence Estimates of ANA, Asthma, and Persistent Infections over 25 years from the National Health and Nutrition Examination Survey, 1988-2012.

positivity varied by household size. In 1999-2000, ANA positives were more likely to be born in the U.S. than ANA negative individuals.

Overall, $17 \%$ of the sample reported an asthma diagnosis, with the prevalence steadily increasing over time (Figure 2). Table 2 shows the adjusted prevalence ORs and 95\% CIs for assessing ANA associations with asthma and allergy by time period and across all time periods combined. "Ever" asthma diagnosis was not associated with ANA positivity in any individual time period, however, OR estimates were elevated in 1999-2000 and 2003-2004. Overall, individuals with an asthma diagnosis had 1.46 times the adjusted odds of ANA positivity as individuals who had never been diagnosed with asthma (95\% CI: $0.84,2.54)$, though this effect estimate was not statistically significant at the 0.05 level.

Of those reporting ever having asthma and a diagnosis age $(n=400), 53 \%$ were diagnosed before the age of 6 years and the proportion of ANA positive individuals who were diagnosed with asthma before age 6 increased from $40 \%$ in 1999-2000 to $74 \%$ in 2011-2012 (Table 1). Individuals with an asthma diagnosis before the age of 6 had 2.07 times the adjusted odds of ANA positivity as those without asthma (95\% CI: 1.08, 3.99) (Table 2). This association was robust to additional adjustment for smoking, country of birth, and household size. No association was observed between asthma diagnosed at age 6 or later and ANA positivity. Approximately $10 \%$ of participants reported current asthma in the full sample, and no consistent associations with ANA positivity were observed.

Self-report of hay fever, representing allergy, was present in $11 \%$ of adolescents overall. In all time periods except 1988-1991, a higher proportion of ANA positive individuals reported hay fever than ANA negative individuals, reaching 21\% in 2011-2012 (Table 1). Overall, individuals with hay fever had 1.55 times the adjusted odds of ANA positivity as those without hay fever, although the 95\% confidence interval contains the null value (95\% CI: 0.85, 2.84, Table 2). The largest period-specific OR was 2.57 (95\% CI: 0.89, 7.74) in 2003-2004.

Few adolescents in this study reported both asthma and hay fever (i.e., "atopic asthma" 2.8\%), and most experienced neither (81.6\%) (Table 1). There were no observed differences by sex (Supplemental Table 1). In 2003-2004, those with atopic asthma had 5.6 times the adjusted odds of ANA positivity as adolescents with neither asthma nor hay fever (95\% CI: 1.05, 29.83) (Table 2). This association was robust to additional adjustment for country of birth, household size, and smoking.

H. pylori seroprevalence decreased from 1988-1991 to 19992000 from $34 \%$ to $26 \%$ among adolescents with ANA results. Among all adolescents assayed for $H$. pylori, regardless of ANA testing, seropositivity decreased from $28 \%$ to $16 \%$ (Figure 2). A greater proportion of male adolescent ANA positive individuals were $H$. pylori seropositive than females in 1988-1991, but this was not observed in 1999-2000. H. pylori in adolescents was inversely associated with ANA positivity (overall OR: 0.49, 95\% CI: $0.24,0.99)$, with the stronger association observed in 19992000 (OR: 0.33, 95\% CI: 0.14, 0.78) (Table 2).

T. gondii seroprevalence decreased over time from $17 \%$ in 1988-1991 to 2.5\% in 2011-2012 (Figure 2). Decreasing frequency of $T$. gondii by ANA status was observed with just under $15 \%$ of ANA positives also being $T$. gondii seropositive in 1988-1991 decreasing to about 3\% in 2011-2012. Overall, adolescents who were seropositive for T. gondii had 1.86 higher adjusted odds of ANA positivity than seronegative adolescents (95\% CI: 0.88, 3.93), and all time periods except 1988-1991 had elevated ORs (Table 2).

In adolescents aged 14-19, HSV-1 seroprevalence was 39\% overall. No statistically significant difference in HSV-1 prevalence by ANA status was observed in any of the time periods, though seroprevalence among ANA positives increased from $24 \%$ in $1988-1991$ to $45 \%$ in $1999-2000$, declining to $32 \%$ in 2003-2004 and 2011-2012. HSV-1 seropositivity was inversely associated with ANA in 1988-1991 (OR: 0.19, 95\% CI 0.07, 0.52) but this association was not statistically significant in other time periods.

\subsection{Time Trend Analyses}

The adjusted odds of ANA positivity increased by approximately $5 \%$ per year in U.S. adolescents from 1988-1991 through 20112012 (trend p-value $<0.001$; Table 2). This effect estimate was largely unchanged by additional adjustment for any of the exposures considered in this study, meaning they did not statistically explain the observed time trend. Since $H$. pylori seropositivity data were only available in 1988-1991 and 19992000, that infection was excluded from trend analysis.

In assessing the relative risk of ANA positivity in each time period individually compared to 1988-1991 (referent), the adjusted odds of ANA positivity in adolescents over time increased from 1.57 (95\% CI: $0.75,3.28)$, to 2.71 (95\% CI: 1.47 , 5.00), and 3.07 (95\% CI: 1.74, 5.42) in 1999-2000, 2003-2004, and 2011-2012, respectively (Figure 3), replicating previous analyses (1). Additional adjustment for country of birth, household size, and smoking did not meaningfully alter these results. 
TABLE 1 | Participant Characteristic Counts and Weighted Proportions by Time Period and ANA Status in U.S. Adolescents.

\begin{tabular}{|c|c|c|c|c|c|c|c|c|c|c|c|c|c|}
\hline \multirow[t]{3}{*}{ Characteristic } & \multicolumn{2}{|c|}{$\begin{array}{c}\text { ANA Status NHANES } \\
1988-1991\end{array}$} & & \multicolumn{3}{|c|}{ ANA Status NHANES 1999-2000 } & \multicolumn{3}{|c|}{ ANA Status NHANES 2003-2004 } & \multicolumn{3}{|c|}{ ANA Status NHANES 2011-2012 } & \multirow{3}{*}{$\begin{array}{c}\text { Overall } \\
\text { ( } \mathrm{N}=2709) \\
\mathrm{n}(\%)\end{array}$} \\
\hline & $\begin{array}{c}\text { Negative } \\
(\mathrm{n}=631,95.0 \%)\end{array}$ & $\begin{array}{c}\text { Positive } \\
(n=45,5.0 \%)\end{array}$ & & 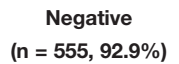 & $\begin{array}{c}\text { Positive } \\
\text { (n= 54, 7.1\%) }\end{array}$ & & $\begin{array}{c}\text { Negative } \\
(n=521,88.4 \%)\end{array}$ & $\begin{array}{c}\text { Positive } \\
\text { (n=60, 11.6\%) }\end{array}$ & & $\begin{array}{c}\text { Negative } \\
(n=741,87.2 \%)\end{array}$ & $\begin{array}{c}\text { Positive } \\
(n=102,12.8 \%)\end{array}$ & & \\
\hline & $n(\%)$ & n (\%) & P-value & $n(\%)$ & $n(\%)$ & $P$-value & n (\%) & n (\%) & P-value & n (\%) & n (\%) & P-value & \\
\hline \multirow{2}{*}{\multicolumn{14}{|c|}{ Demographics }} \\
\hline \multicolumn{6}{|l|}{ Sex } & & & & & & & & \\
\hline Female & $325(48.1)$ & $35(67.8)$ & 0.086 & 257 (45.2) & $36(66.1)$ & 0.034 & $239(48.1)$ & 46 (73.5) & 0.015 & $349(47.8)$ & 60 (55.9) & 0.284 & $1347(49.0)$ \\
\hline \multicolumn{14}{|l|}{$\begin{array}{l}\text { Male } \\
\text { Age (Years) }\end{array}$} \\
\hline $12-13$ & $161(24.1)$ & $7(27.0)$ & 0.437 & $153(23.0)$ & $18(22.9)$ & 0.896 & $133(27.8)$ & $9(13.2)$ & 0.193 & $186(24.9)$ & $28(27.5)$ & 0.719 & $695(24.7)$ \\
\hline $14-15$ & $126(18.1)$ & $17(24.8)$ & & $123(24.1)$ & $12(27.7)$ & & $122(23.8)$ & $15(26.6)$ & & $186(26.0)$ & $19(26.8)$ & & 620 (23.6) \\
\hline $16-17$ & $175(29.0)$ & $13(36.9)$ & & $145(25.1)$ & $8(27.6)$ & & $136(25.1)$ & $19(39.0)$ & & $178(24.1)$ & $26(17.7)$ & & $700(26.0)$ \\
\hline $18-19$ & $169(28.8)$ & $8(11.2)$ & & $134(27.8)$ & $16(21.8)$ & & $130(23.2)$ & $17(21.2)$ & & $191(25.0)$ & $29(28.1)$ & & $694(25.7)$ \\
\hline \multicolumn{14}{|l|}{ Race/Ethnicity } \\
\hline White & $202(67.9)$ & $9(57.4)$ & 0.298 & $116(57.2)$ & $11(54.2)$ & 0.239 & $136(64.8)$ & $16(67.4)$ & 0.871 & $167(54.4)$ & $27(63.2)$ & 0.102 & $684(61.1)$ \\
\hline Black & 177 (15.5) & $9(14.2)$ & & $138(12.8)$ & $18(22.5)$ & & $192(15.7)$ & 20 (12.5) & & 219 (15.3) & $31(15.1)$ & & $804(14.8)$ \\
\hline \multirow{2}{*}{\multicolumn{14}{|c|}{ Body Mass Inc }} \\
\hline & & & & & & & & & & & & & \\
\hline Underweight/Normal & $470(78.0)$ & 34 (76.6) & 0.963 & $330(67.7)$ & $35(81.2)$ & 0.062 & $296(56.1)$ & $46(83.8)$ & 0.001 & $449(63.1)$ & $69(69.4)$ & 0.405 & $1729(66.3)$ \\
\hline $\begin{array}{l}\text { Overweight } \\
\text { Obese }\end{array}$ & $\begin{array}{l}72(10.1) \\
89(11.9)\end{array}$ & $\begin{array}{l}7(12.2) \\
4(11.1)\end{array}$ & & $\begin{array}{l}91(14.9) \\
130(17.4)\end{array}$ & $\begin{array}{c}6(6.2) \\
13(12.6)\end{array}$ & & $\begin{array}{c}96(19.5) \\
128(24.3)\end{array}$ & $\begin{array}{l}9(6.0) \\
5(10.2)\end{array}$ & & $\begin{array}{l}113(15.5) \\
164(213)\end{array}$ & & & $\begin{array}{l}411(15.0) \\
546(18.6)\end{array}$ \\
\hline $\begin{array}{l}\text { Obese } \\
\text { Missing }\end{array}$ & $\begin{array}{c}89(11.9) \\
0\end{array}$ & $\begin{array}{c}4(11.1) \\
0\end{array}$ & & $\begin{array}{c}130(1 / .4) \\
4\end{array}$ & 0 & & $128(2.3)$ & $\begin{array}{c}5(1+.2) \\
0\end{array}$ & & $\begin{array}{c}164(21.3) \\
15\end{array}$ & $\begin{array}{l}13(13.9) \\
3\end{array}$ & & \\
\hline \multicolumn{14}{|l|}{ Education of Reference Parent } \\
\hline Less than High School & $292(28.8)$ & $23(31.8)$ & 0.952 & $234(27.3)$ & $24(25.6)$ & 0.979 & $179(20.5)$ & $19(19.8)$ & 0.381 & $198(25.1)$ & $22(13.3)$ & 0.052 & $991(24.6)$ \\
\hline High School Grad/GED or Equivalent & $178(32.7)$ & $10(31.3)$ & & $134(26.8)$ & $11(26.3)$ & & $116(27.1)$ & $12(17.7)$ & & 166 (22.3) & $30(20.2)$ & & $657(26.6)$ \\
\hline Some College or Above & $160(38.5)$ & $11(36.9)$ & & $158(46.0)$ & $16(48.1)$ & & $200(52.4)$ & $27(62.4)$ & & $346(52.7)$ & $49(66.5)$ & & $967(48.8)$ \\
\hline Missing & 1 & 1 & & 29 & 3 & & 26 & 2 & & 31 & 1 & & 94 \\
\hline \multicolumn{14}{|l|}{ Smoking (Cotinine) } \\
\hline Smoking ( $\geq 15 \mathrm{ng} / \mathrm{mL})$ & $96(21.7)$ & $4(3.7)$ & 0.017 & $79(21.7)$ & $7(7.2)$ & 0.170 & $68(16.3)$ & $2(5.6)$ & 0.321 & $64(8.5)$ & $7(6.9)$ & 0.888 & $327(16.2)$ \\
\hline Secondhand (LOD-15ng/mL) & $466(69.8)$ & 35 (88.1) & & $290(55.4)$ & $26(61.9)$ & & $384(71.2)$ & 45 (80.3) & & $461(60.9)$ & $71(61.7)$ & & $1778(64.8)$ \\
\hline Not detected (BLOD) & $50(8.5)$ & $5(8.2)$ & & $178(22.9)$ & $21(30.8)$ & & $69(12.5)$ & $13(14.1)$ & & $216(30.6)$ & $24(31.4)$ & & 576 (18.9) \\
\hline & 19 & 1 & & 8 & 0 & & 0 & 0 & & 0 & 0 & & 28 \\
\hline \multicolumn{14}{|l|}{ Household Size } \\
\hline 1-3 & $136(26.7)$ & $7(29.9)$ & 0.171 & $120(31.7)$ & $16(35.8)$ & 0.459 & $130(29.4)$ & $12(7.6)$ & $<0.001$ & $185(25.6)$ & $21(26.7)$ & 0.251 & $627(28.1)$ \\
\hline $4-5$ & $304(52.4)$ & 20 (36.6) & & $241(47.6)$ & $23(53.0)$ & & $246(52.0)$ & 35 (83.4) & & $382(53.1)$ & $58(61.3)$ & & $1309(52.4)$ \\
\hline \multirow{2}{*}{\multicolumn{14}{|c|}{ Birth Country }} \\
\hline & & & & & & & & & & & & & \\
\hline United States & $523(89.0)$ & $34(93.2)$ & 0.361 & $451(88.8)$ & $48(97.0)$ & 0.008 & $453(91.0)$ & $52(87.3)$ & 0.565 & $617(89.4)$ & 94 (93.5) & 0.251 & $2273(89.8)$ \\
\hline $\begin{array}{l}\text { Other } \\
\text { Missing }\end{array}$ & $103(11.0)$ & $11(6.8)$ & & $104(11.2)$ & $6(3.0)$ & & $68(9.0)$ & $8(12.7)$ & & $124(10.6)$ & $8(6.5)$ & & $432(10.2)$ \\
\hline $\begin{array}{l}\text { Missing } \\
\text { Infection Seropositivity }\end{array}$ & 4 & 0 & & 0 & 0 & & 0 & 0 & & 0 & 0 & & 4 \\
\hline \multicolumn{14}{|l|}{ Infection Seropositivity } \\
\hline Helicobacter pylori & $212(29.0)$ & $15(23.6)$ & 0.588 & $150(16.6)$ & $10(7.9)$ & 0.111 & & & & & & & \\
\hline Toxoplasma gondii & $112(18.1)$ & $5(14.7)$ & 0.669 & $57(7.0)$ & $5(16.9)$ & 0.127 & $29(7.1)$ & $4(11.2)$ & 0.427 & $21(2.3)$ & $5(3.2)$ & 0.524 & $238(8.4)$ \\
\hline Herpes Simplex Virus- $1^{\text {a }}$ & $251(45.1)$ & $20(24.3)$ & 0.057 & $216(40.6)$ & $21(44.5)$ & 0.686 & 185 (38.6) & $21(31.9)$ & 0.394 & $222(35.1)$ & $33(32.0)$ & 0.584 & $969(39.1)$ \\
\hline Health Status & & & & & & & & & & & & & \\
\hline Ever asthma diagnosis & $52(7.9)$ & $1(1.8)$ & 0.076 & $71(12.7)$ & $6(18.1)$ & 0.531 & $86(19.2)$ & $17(27.0)$ & 0.322 & $156(24.1)$ & $24(26.7)$ & 0.599 & $413(16.8)$ \\
\hline Current asthma & $38(5.7)$ & $1(1.8)$ & 0.191 & $46(8.2)$ & $3(7.4)$ & 0.899 & $56(10.4)$ & $12(17.0)$ & 0.379 & $90(14.7)$ & $13(13.1)$ & 0.801 & $259(10.0)$ \\
\hline Asthma diagnosis age $(n=400)$ & & & & & & & & & & & & & \\
\hline$<6$ years old & $26(59.3)$ & $0(0.0)$ & NA & $30(39.9)$ & $3(39.8)$ & 0.998 & $46(54.2)$ & $10(77.4)$ & 0.084 & $85(51.3)$ & $10(74.0)$ & 0.019 & $210(52.6)$ \\
\hline $\begin{array}{l}6 \text { years or older } \\
\text { Current hav fever }\end{array}$ & $20(40.7)$ & 1 (100.) & & $41(60.1)$ & $3(60.2)$ & & $39(45.8)$ & 6 (22.6) & & $68(48.7)$ & $12(26.0)$ & & $190(47.4)$ \\
\hline $\begin{array}{l}\text { Current hay fever } \\
\text { Atopic Asthma }\end{array}$ & $49(10.3)$ & $3(4.2)$ & 0.157 & $60(10.1)$ & $9(13.0)$ & 0.545 & $44(9.9)$ & $6(18.1)$ & 0.158 & $83(13.0)$ & $17(20.9)$ & 0.304 & $271(11.2)$ \\
\hline $\begin{array}{l}\text { Atopic Asthma } \\
\text { Current asthma and hay fever }\end{array}$ & $8(1.5)$ & $0(0.0)$ & NA & $13(2.5)$ & $1(1.1)$ & 0.778 & $11(2.3)$ & $2(4.3)$ & 0.556 & $21(4.6)$ & $2(5.3)$ & 0.634 & $58(2.8)$ \\
\hline Current hay fever only & $41(8.7)$ & $3(4.2)$ & & $46(7.5)$ & $8(11.9)$ & & $33(7.6)$ & $4(13.8)$ & & $62(8.4)$ & 15 (15.6) & & $212(8.4)$ \\
\hline Current asthma only & $30(4.2)$ & $1(1.8)$ & & $33(5.8)$ & $2(6.4)$ & & $45(8.2)$ & $10(12.7)$ & & $68(9.7)$ & $11(7.7)$ & & $200(7.2)$ \\
\hline No atopy & $551(85.5)$ & $41(94.0)$ & & $459(84.2)$ & $42(80.6)$ & & $431(81.9)$ & $44(69.2)$ & & $584(77.3)$ & $74(71.3)$ & & $2226(81.6)$ \\
\hline
\end{tabular}

${ }^{a}$ Ages 14-19 Only. 


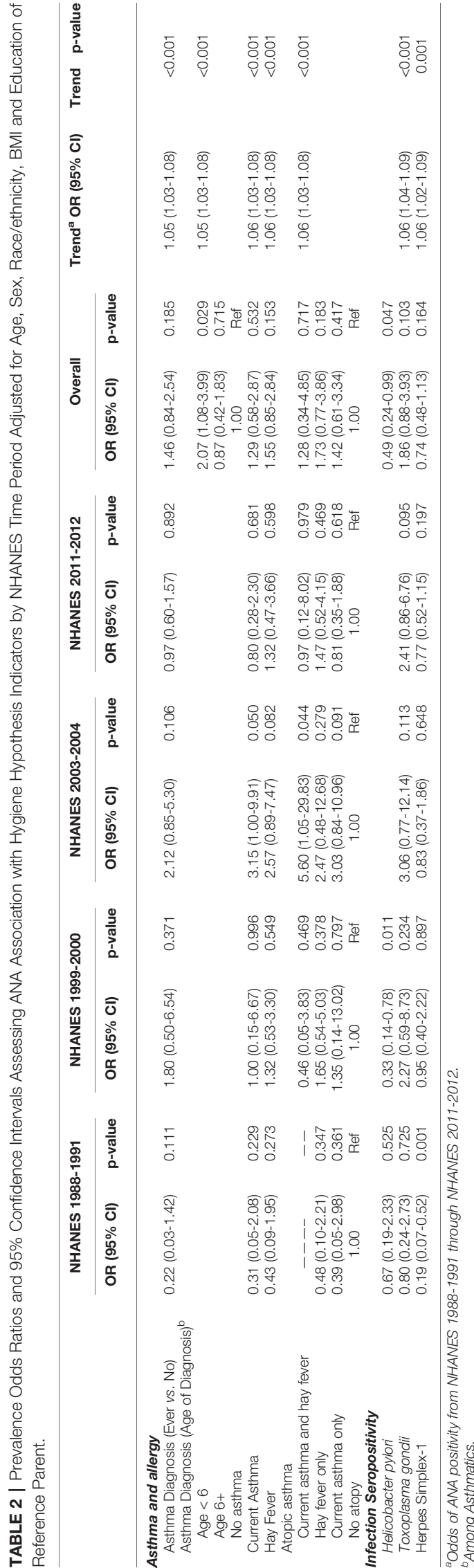

\subsection{Additional Analyses}

Age-adjusted prevalence ORs and 95\% CIs for the association between potential model covariates and ANA positivity by time period and overall are reported in Supplemental Table 2. We also looked at ANA staining patterns, but no clear time trends were seen in the proportion of patterns (Supplemental Table 3). Compared to a NHANES sample including adolescents and adults (i.e. ages 12+) (1), in our study of adolescents only, periods 1 and 3 show a higher proportion had dense fine speckled and total mitotic staining, and we saw a lower proportion with cytoplasmic staining across all periods. In addition, we examined elevated ANA staining intensity (3 or 4 +) by time period and found that the 2011-2012 time period had a higher proportion of adolescents with 3 or $4+$ relative to the other time periods (Supplemental Table 3). However, due to a small sample size, this should be replicated in a larger sample.

\section{DISCUSSION}

ANA prevalence has steadily increased in U.S. adolescents over a recent 25-year span (1). Adolescence is a time of immense change in physical growth and development. In particular, immune system development extends from the prenatal period through adolescence (12), and immune competence continually builds as neoantigens are encountered (13). Due to the pace of growth and hormonal shifts, late childhood and adolescence is a sensitive period where adolescents are particularly vulnerable to environmental exposures (13). The observed increase in ANA among adolescents, a 200\% increase in the odds of ANA in 201112 compared to 1988-1991, is alarming and may represent temporal shifts in environmental exposures for this age range. We investigated whether the increase in adolescent ANA prevalence correlated with other factors associated with improved hygiene and cleaner environments in childhood, consistent with the hygiene hypothesis. Overall, our findings were mixed. Increases in ANA prevalence over time did not appear to be explained by asthma, allergy, or the infections that we studied.

However, we did observe a positive association between asthma diagnosis before 6 years and ANA positivity. It is possible that the airway inflammation (14) characteristic of this asthma phenotype and ANA may have a common underlying cause, including early life exposures. The co-occurrence of ANA and early-onset asthma may become more common in U.S. adolescents, though additional data from more proximal time periods is needed to monitor for a potential trend. Understanding the pathophysiological pathways that may link early-onset asthma and biomarkers of autoimmunity will be an important area of future research.

Though we did not observe consistent inverse relationships between the infections examined and ANA, as would be expected under the hygiene hypothesis, the data did suggest an inverse association between $H$. pylori and ANA positivity. H. pylori was previously shown to be protective for early-onset (less than 5 years) childhood asthma in NHANES (15). H. pylori reduces host inflammatory responses, even early in life, which in animal 


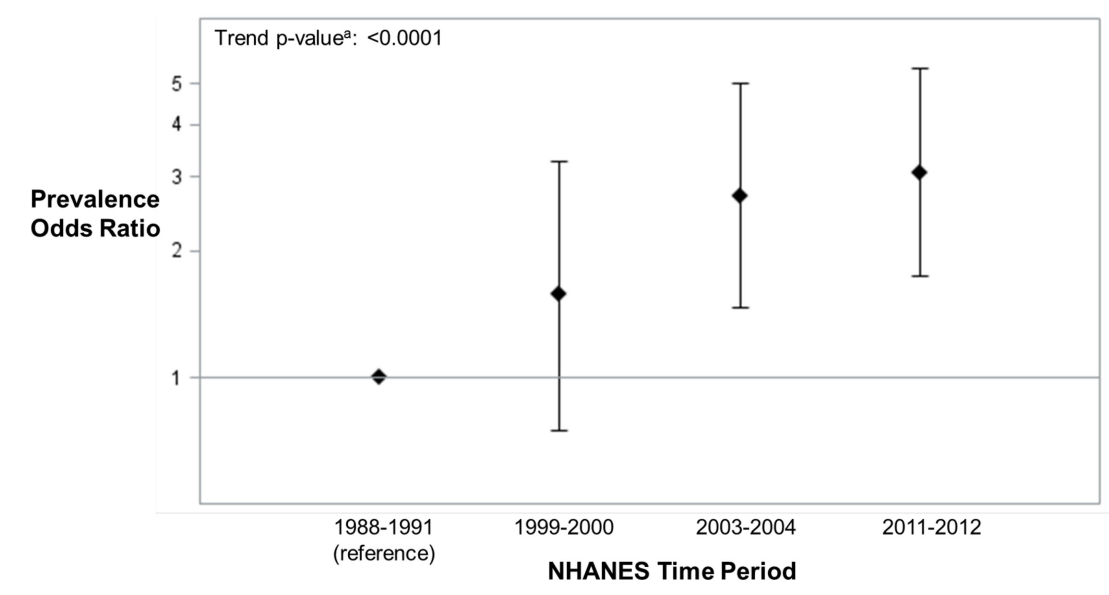

FIGURE 3 | ANA Prevalence Odds Ratios (95\% Confidence Intervals) in U.S. Adolescents by NHANES Time Period (n=2594) adjusted for age, sex, race/ethnicity, BMl

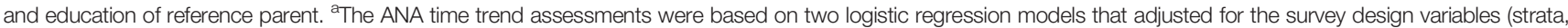
clusters, and sampling weights) and categorical covariates. One model added a categorical covariate for time period and estimated the ANA prevalence odds ratio for each period, relative to the first. The other model added a quantitative covariate for the number of years between period midpoints, relative to the first, and produced a P-value from a $\chi^{2}$-test to assess an ANA time trend.

models have been shown to provide protective effects against allergic airway inflammation in first and second generation offspring (16-18). As with other persistent pathogens, the early life benefits of $H$. pylori infection may wane and undermine the mature adult immune system, resulting in detrimental outcomes (19-21). Indeed, a recent study of $H$. pylori in U.S. adults aged $20+$ years found that $H$. pylori seropositivity was associated with higher odds of ANA positivity, suggesting that chronic inflammation resulting from $H$. pylori may impact immunological regulation (22). Additional research is needed to understand the relationship of persistent pathogens, such as $H$. pylori, and immune self-tolerance over the life course.

Elevated odds of ANA were observed for adolescents with atopic asthma in one time period (2003-2004), but these results were not replicated in other periods. Overall, atopic asthma was rare in adolescents $(2.8 \%)$, so analyses were underpowered. Previous research on atopic asthma and autoimmune disease, in part motivated by the idea of antagonism between allergic and autoimmune responses, has shown mixed results spanning positive, negative, and no associations, with heterogenous definitions of autoimmune disease and atopy (23). Crossregulatory T-helper Type 1 (TH1) versus Type 2 (TH2) immune responses have been hypothesized to lead to negative relationships or antagonism between allergic and autoimmune diseases $(24,25)$. According to the contemporary hygiene hypothesis, cleaner environments and less exposure to infections provide inadequate education of the immune system, resulting in higher likelihood of allergic and autoimmune disease. Thus, we would expect the occurrence of atopic asthma and autoimmunity to be correlated (26). Whether the co-occurrence is due to the same underlying dysregulation of immunological processes is an area of active research. Studies investigating atopic dermatitis in children have shown increased risk for autoimmune diseases (27). Studies have found positive associations between IgE autoantibodies and atopic dermatitis in children (26), and that children with atopic dermatitis develop ANA earlier than children without atopic dermatitis (27), suggesting potential for an underlying common cause including autoreactive T-cells and molecular mimicry (26). Further research on atopic asthma and autoimmunity is needed to understand common underlying mechanisms.

Our study has several limitations. We were limited to selfreported allergy, asthma and atopy information because NHANES did not link to participant medical records. Further, we do not have information on family history of autoimmune disorders because this was not asked in the NHANES surveys. Some of the conditions and infections examined had low prevalence, reducing power and precision of statistical analyses. Other infections examined in studies of the hygiene hypothesis, such as Hepatitis A (28), were not included due to vaccine licensure and varying vaccine updates during the study period. Previous studies have shown that ANA are more commonly detected during infections, and though autoimmunity may have a positive role during certain infections (29), in our study, ANA were not associated with self-reported infection (head or chest cold) in the last four weeks. The serological data in NHANES are cross-sectional so we cannot determine the temporal relationships of infections with ANA incidence. Longitudinal studies are warranted to better understand the age at onset and persistence of ANA, particularly in existing pediatric cohorts including biomarkers related to allergy and asthma phenotype (30). Detection of ANA by immunofluorescence identifies a wide range of autoantibodies and staining patterns. While we did not have data on diseasespecific autoantibodies, these are uncommon in the general population $(<10 \%)(31)$. The clinical interpretation of specific patterns is an important area of ongoing research. There is little known about ANA patterns in children and the natural history of 
ANA over time as they age into adulthood, though studies suggest varying factors, such as genetic variability and immune phenotype may be related to risk of developing autoimmune diseases (32). Investigation of specific cellular patterns with atopy is an important area for future research.

Despite these limitations, our study has strengths. We used nationally representative data spanning 25 years to understand increasing prevalence of ANA in U.S. adolescents. We contrasted ANA and serostatus to several infections whereas previous studies only looked at the association between ANA and infections among ANA positive individuals (33). NHANES is designed to examine national trends in health over time and the infection assays are of high quality and comparable over time. Further, we were able to control for several important variables in examining drivers of hygiene hypothesis exposures, including age, sex, race/ethnicity, BMI, and education. In sensitivity analyses, we additionally controlled for country of birth, smoking status and household size, as these factors may influence exposure to hygiene hypothesis indicators, but the addition of these potential confounders did not meaningfully change effect estimates. Our previous work has shown that vitamin $\mathrm{D}$ deficiency was associated with ANA positivity in older US adults (aged 50+) (34). In the current study, however, no statistically significant differences in vitamin D levels by ANA status were observed, therefore it was not considered as a confounder. Our previous work (35) also found that some medications were associated with ANA in the general adult $(18+)$ population, including bronchodilators in those ages 60 and older, but medication use and ANA in adolescents was not examined. A thorough examination of medication use goes beyond the scope of the current study, but is an important area of future research.

Though the results from our study were mixed, ANA may be an additional indicator of inadequate immune system education in childhood and adolescence. The immunology of the hygiene hypothesis is complex with multiple mechanisms potentially driving associations. Proper immune maturation requires antigenically rich environments; the greater frequency of selftolerance loss in younger age groups observed in our study may be the result of reduced microbiome diversity, poor immunologic education, and molecular mimicry $(29,36)$. Characterizing more recent birth cohorts by ANA status, as well as allergy, atopic asthma, and infections will be an important next step in determining if trends observed in this study are maintained or diminished. In addition, as the birth cohorts examined in this study enter mid-life, monitoring for increases in autoimmune disease will shed light on the connection between early

\section{REFERENCES}

1. Dinse GE, Parks CG, Weinberg CR, Co CA, Wilkerson J, Zeldin DC, et al. Increasing Prevalence of Antinuclear Antibodies in the United States. Arthritis Rheumatol (2020) 72(6):1026-35. doi: 10.1002/art.41214

2. Rook GA, Raison CL, Lowry CA. Microbial 'Old Friends', Immunoregulation and Socioeconomic Status. Clin Exp Immunol (2014) 177(1):1-12. doi: 10.1111/cei.12269 appearance of autoimmunity markers and autoimmune disease risk. Early ANA occurrence may also be a sentinel for poor immunological regulation and cellular aging is associated with ANA incidence, therefore, future studies should investigate whether children with ANA have more aged immune systems than their chronological age may represent.

\section{DATA AVAILABILITY STATEMENT}

Publicly available datasets were analyzed in this study. This data can be found here: https://www.cdc.gov/nchs/nhanes/index.htm.

\section{ETHICS STATEMENT}

The studies involving human participants were reviewed and approved by National Center for Health Statistics (NCHS) Ethics Review Board of the Centers for Disease Control and Prevention (CDC). Written informed consent to participate in this study was provided by the participants' legal guardian/next of kin.

\section{AUTHOR CONTRIBUTIONS}

Conceived of and designed the study: HM, CP, and DS. Acquired the data and performed the analysis: JW. Drafted the manuscript: HM and JW. Consulted on analytic strategy and critically revised manuscript: CP, DS, FM, and GD. All authors contributed to the article and approved the submitted version.

\section{FUNDING}

This work was supported in part by the Intramural Research Program of the NIH, National Institute of Environmental Health Sciences (Z01-ES049028) and contract HHSN273201600011C to Social \& Scientific Systems, and in part by the Shaw Scientist Award from the Greater Milwaukee Foundation.

\section{SUPPLEMENTARY MATERIAL}

The Supplementary Material for this article can be found online at: https://www.frontiersin.org/articles/10.3389/fimmu.2022. 789379/full\#supplementary-material 
5. Strachan DP. Hay Fever, Hygiene, and Household Size. BMJ (1989) 299 (6710):1259-60. doi: 10.1136/bmj.299.6710.1259

6. Strachan DP. Family Size, Infection and Atopy: The First Decade of the "Hygiene Hypothesis". Thorax (2000) 55 Suppl 1:S2-10. doi: 10.1136/thorax.55.suppl_1.S2

7. Jatzlauk G, Bartel S, Heine H, Schloter M, Krauss-Etschmann S. Influences of Environmental Bacteria and Their Metabolites on Allergies, Asthma, and Host Microbiota. Allergy (2017) 72(12):1859-67. doi: 10.1111/all.13220

8. Fanfair RN, Zaidi A, Taylor LD, Xu F, Gottlieb S, Markowitz L. Trends in Seroprevalence of Herpes Simplex Virus Type 2 Among non-Hispanic Blacks and non-Hispanic Whites Aged 14 to 49 Years-United States, 1988 to 2010. Sex Transm Dis (2013) 40(11):860-4. doi: 10.1097/OLQ.0000000000000043

9. Kruszon-Moran D, McQuillan G, Kim-Farley R. Prevalence, Change Over Time, and Comparison With U.S. Estimates of Selected Infectious Diseases in Los Angeles County: Findings From the National Health and Nutrition Examination Survey, 1999-2006 and 2007-2014. Natl Health Stat Rep (2018) 119):1-17.

10. Johnson CL, Paulose-Ram R, Ogden CL, Carroll MD, Kruszon-Moran D, Dohrmann SM, et al. National Health and Nutrition Examination Survey: Analytic Guidelines, 1999-2010. Vital Health Stat 2 (2013) 161):1-24.

11. Center for Disease Control and Prevention. National Health and Nutrition Examination Survey 2011-2012 Data Documentation, Codebook, and Frequencies Herpes Simplex Virus Type-1 \& Type-2 (HSV_G) (2013). Available at: https://wwwn.cdc.gov/nchs/nhanes/2011-2012/HSV_G.htm.

12. Georgountzou A, Papadopoulos NG. Postnatal Innate Immune Development: From Birth to Adulthood. Front Immunol (2017) 8(957). doi: 10.3389/ fimmu.2017.00957

13. West LJ. Defining Critical Windows in the Development of the Human Immune System. Hum Exp Toxicol (2002) 21(9-10):499-505. doi: 10.1191/ 0960327102ht288oa

14. Trivedi M, Denton E. Asthma in Children and Adults-What Are the Differences and What Can They Tell Us About Asthma? Front Pediatr (2019) 7:256. doi: 10.3389/fped.2019.00256

15. Chen Y, Blaser MJ. Helicobacter Pylori Colonization is Inversely Associated With Childhood Asthma. J Infect Dis (2008) 198(4):553-60. doi: 10.1086/ 590158

16. Altobelli A, Bauer M, Velez K, Cover TL, Muller A. Helicobacter Pylori VacA Targets Myeloid Cells in the Gastric Lamina Propria To Promote Peripherally Induced Regulatory T-Cell Differentiation and Persistent Infection. mBio (2019) 10(2):e00261-19. doi: 10.1128/mBio.00261-19

17. Kyburz A, Fallegger A, Zhang X, Altobelli A, Artola-Boran M, Borbet T, et al. Transmaternal Helicobacter Pylori Exposure Reduces Allergic Airway Inflammation in Offspring Through Regulatory T Cells. J Allergy Clin Immunol (2019) 143(4):1496-512 el1. doi: 10.1016/j.jaci.2018.07.046

18. Lehours P, Ferrero RL. Review: Helicobacter: Inflammation, Immunology, and Vaccines. Helicobacter (2019) 24 Suppl 1:e12644. doi: 10.1111/hel.12644

19. Aiello AE, Haan MN, Pierce CM, Simanek AM, Liang J. Persistent Infection, Inflammation, and Functional Impairment in Older Latinos. J Gerontol Ser A Biol Sci Med Sci (2008) 63(6):610-8. doi: 10.1093/gerona/63.6.610

20. Barnes LL, Capuano AW, Aiello AE, Turner AD, Yolken RH, Torrey EF, et al. Cytomegalovirus Infection and Risk of Alzheimer Disease in Older Black and White Individuals. J Infect Dis (2015) 211(2):230-7. doi: 10.1093/infdis/jiu437

21. Simanek AM, Dowd JB, Pawelec G, Melzer D, Dutta A, Aiello AE. Seropositivity to Cytomegalovirus, Inflammation, All-Cause and Cardiovascular Disease-Related Mortality in the United States. PloS One (2011) 6(2):e16103. doi: 10.1371/journal.pone.0016103

22. Meier HCS, Miller FW, Dinse GE, Weinberg CR, Cho CC, Parks CG. Helicobacter Pylori Seropositivity is Associated With Antinuclear Antibodies in US Adults, NHANES 1999-2000. Epidemiol Infect (2020) 148: e20. doi: $10.1017 /$ S0950268820000126

23. Rabin RL, Levinson AI. The Nexus Between Atopic Disease and Autoimmunity: A Review of the Epidemiological and Mechanistic
Literature. Clin Exp Immunol (2008) 153(1):19-30. doi: 10.1111/j.13652249.2008.03679.x

24. Wynn TA. Type 2 Cytokines: Mechanisms and Therapeutic Strategies. Nat Rev Immunol (2015) 15(5):271-82. doi: 10.1038/nri3831

25. Lindelof B, Granath F, Tengvall-Linder M, Lindelof H, Ekbom A. Allergy and Autoimmune Disease: A Registry-Based Study. Clin Exp Allergy (2009) 39 (1):110-5. doi: 10.1111/j.1365-2222.2008.03115.x

26. Bach JF. The Effect of Infections on Susceptibility to Autoimmune and Allergic Diseases. N Engl J Med (2002) 347(12):911-20. doi: 10.1056/ NEJMra020100

27. Kokkonen J, Niinimaki A. Increased Incidence of Autoimmune Disorders as a Late Complication in Children With Early Onset Dermatitis and/or Milk Allergy. J Autoimmun (2004) 22(4):341-4. doi: 10.1016/j.jaut.2004.03.006

28. Matricardi PM, Rosmini F, Panetta V, Ferrigno L, Bonini S. Hay Fever and Asthma in Relation to Markers of Infection in the United States. J Allergy Clin Immunol (2002) 110(3):381-7. doi: 10.1067/mai.2002.126658

29. Rivera-Correa J, Rodriguez A. Divergent Roles of Antiself Antibodies During Infection. Trends Immunol (2018) 39(7):515-22. doi: 10.1016/j.it.2018.04.003

30. Klos M, Thurmann L, Bauer M, Heinrich J, Standl M, von Berg A, et al. Longitudinal Trends of Serum IgE and IL5RA Expression Throughout Childhood are Associated With Asthma But Not With Persistent Wheeze. Allergy (2019) 74(10):2002-6. doi: 10.1111/all.13837

31. Satoh M, Chan EK, Ho LA, Rose KM, Parks CG, Cohn RD, et al. Prevalence and Sociodemographic Correlates of Antinuclear Antibodies in the United States. Arthritis Rheum (2012) 64(7):2319-27. doi: 10.1002/art.34380

32. Pisetsky DS. Immune Phenotypes in Individuals Positive for Antinuclear Antibodies: The Impact of Race and Ethnicity. J Allergy Clin Immunol (2020) 146(6):1346-8. doi: 10.1016/j.jaci.2020.09.006

33. Im JH, Chung MH, Park YK, Kwon HY, Baek JH, Lee SY, et al. Antinuclear Antibodies in Infectious Diseases. Infect Dis (Lond) (2020) 52(3):177-85. doi: 10.1080/23744235.2019.1690676

34. Meier HC, Sandler DP, Simonsick EM, Parks CG. Association Between Vitamin D Deficiency and Antinuclear Antibodies in Middle-Aged and Older U.S. Adults. Cancer Epidemiol Biomarkers Prev: Publ Am Assoc Cancer Res Cosponsored Am Soc Prev Oncol (2016) 25(12):1559-63. doi: 10.1158/1055-9965.EPI-16-0339

35. Dinse GE, Parks CG, Meier HCS, Co CA, Chan EKL, Jusko TA, et al. Prescription Medication Use and Antinuclear Antibodies in the United States, 1999-2004. J Autoimmun (2018) 92:93-103. doi: 10.1016/ j.jaut.2018.05.006

36. Lambrecht BN, Hammad H. The Immunology of the Allergy Epidemic and the Hygiene Hypothesis. Nat Immunol (2017) 18(10):1076-83. doi: 10.1038/ ni. 3829

Conflict of Interest: The authors declare that the research was conducted in the absence of any commercial or financial relationships that could be construed as a potential conflict of interest.

Publisher's Note: All claims expressed in this article are solely those of the authors and do not necessarily represent those of their affiliated organizations, or those of the publisher, the editors and the reviewers. Any product that may be evaluated in this article, or claim that may be made by its manufacturer, is not guaranteed or endorsed by the publisher.

Copyright (๑) 2022 Meier, Sandler, Wilkerson, Miller, Dinse and Parks. This is an open-access article distributed under the terms of the Creative Commons Attribution License (CC BY). The use, distribution or reproduction in other forums is permitted, provided the original author(s) and the copyright owner(s) are credited and that the original publication in this journal is cited, in accordance with accepted academic practice. No use, distribution or reproduction is permitted which does not comply with these terms. 